\title{
Resonant switch model of twin peak HF QPOs applied to the atoll source 4U 1636-53
}

\author{
Zdeněk Stuchlík, Andrea Kotrlová and Gabriel Török
}

\author{
Institute of Physics, Faculty of Philosophy and Science, Silesian University in Opava, \\ Bezručovo nám. 13, CZ-74601 Opava, Czech Republic \\ email: zdenek.stuchlik@fpf.slu.cz, andrea.kotrlova@fpf.slu.cz
}

\begin{abstract}
We present a Resonant Switch (RS) model of twin peak high-frequency quasi-periodic oscillations (HF QPOs), assuming switch of twin oscillations at a resonant point, where frequencies of the upper and lower oscillations $\nu_{\mathrm{U}}$ and $\nu_{\mathrm{L}}$ become to be commensurable and the twin oscillations change from one pair of the oscillating modes (corresponding to a specific model of HF QPOs) to some other pair due to non-linear resonant phenomena. The RS model enables to determine range of allowed values of spin $a$ and mass $M$ of the neutron star located at the atoll source $4 \mathrm{U} 1636-53$ where two resonant points are observed at frequency ratios $\nu_{\mathrm{U}}: \nu_{\mathrm{L}}=3: 2$, $5: 4$.
\end{abstract}

Keywords. stars: neutron - X-rays: binaries — accretion, accretion disks

\section{Introduction}

In the case of LMXBs containing neutron or quark stars we propose a new model of twin peak HF QPOs assuming switching of the twin oscillatory modes creating sequences of the lower and upper HF QPOs at a resonant point. According to such a Resonant Switch (RS) model non-linear resonant phenomena will cause excitation of a new oscillatory mode (or two new oscillatory modes) and vanishing of one of the previously acting modes (or both the previous modes), i.e., switching from one pair of the oscillatory modes to other pair of them that will be acting up to the following resonant point (Stuchlík et al. 2012).

\section{Resonant switch model of HF QPOs in neutron star systems}

We assume two resonant points at the disc radii $r_{\text {out }}$ and $r_{\text {in }}$, with observed frequencies $\nu_{\mathrm{U}}^{\text {out }}, \nu_{\mathrm{L}}^{\text {out }}$ and $\nu_{\mathrm{U}}^{\text {in }}, \nu_{\mathrm{L}}^{\text {in }}$, being in commensurable ratios $p^{\text {out }}=n^{\text {out }}: m^{\text {out }}$ and $p^{\text {in }}=$ $n^{\text {in }}: m^{\text {in }}$. These resonant frequencies are determined by the energy switch effect (Török 2009); observations put the restrictions $\nu_{\mathrm{U}}^{\text {in }}>\nu_{\mathrm{U}}^{\text {out }}$ and $p^{\text {in }}<p^{\text {out }}$. In the region covering the resonant point at $r_{\text {out }}$ the twin oscillatory modes with the upper (lower) frequency are determined by the function $\nu_{\mathrm{U}}^{\text {out }}(x ; M, a)\left(\nu_{\mathrm{L}}^{\text {out }}(x ; M, a)\right)$. Near the inner resonant point at $r_{\text {in }}$ different oscillatory modes given by the frequency functions $\nu_{\mathrm{U}}^{\text {in }}(x ; M, a)$ and $\nu_{\mathrm{L}}^{\text {in }}(x ; M, a)$ occur $(x \equiv r / M$ is the dimensionless radius $)$.

We assume all the frequency functions to be determined by combinations of the orbital and epicyclic frequencies of the geodesic motion in the Kerr backgrounds. Such a simplification is correct with high precision for neutron (quark) stars with large masses, close to the maximum mass allowed for a given equation of state. The frequency functions have to meet the observationally given resonant frequencies. In the framework of the simple RS model, when two resonant points and two pairs of the frequency functions are assumed, this requirement enables determination of the neutron (quark) star parameters. The "shooting" of the frequency functions to the resonant points can be realized efficiently in two steps. Independence of the frequency ratio on the mass parameter $M$ 
implies the conditions

$$
\nu_{\mathrm{U}}^{\text {out }}(x ; M, a): \nu_{\mathrm{L}}^{\text {out }}(x ; M, a)=p^{\text {out }}, \quad \nu_{\mathrm{U}}^{\text {in }}(x ; M, a): \nu_{\mathrm{L}}^{\text {in }}(x ; M, a)=p^{\text {in }}
$$

giving relations for the spin $a$ in terms of the dimensionless radius $x$ and the resonant frequency ratio $p$. They can be expressed in the form $a^{\text {out }}\left(x, p^{\text {out }}\right)$ and $a^{\text {in }}\left(x, p^{\text {in }}\right)$, or in an inverse form $x^{\text {out }}\left(a, p^{\text {out }}\right)$ and $x^{\text {in }}\left(a, p^{\text {in }}\right)$. At the resonant radii the conditions

$$
\nu_{\mathrm{U}}^{\text {out }}=\nu_{\mathrm{U}}^{\text {out }}(x ; M, a), \quad \nu_{\mathrm{U}}^{\text {in }}=\nu_{\mathrm{U}}^{\text {in }}(x ; M, a)
$$

are satisfied along the functions $M_{p_{\text {out }}}^{\text {out }}(a)$ and $M_{p_{\text {in }}}^{\text {in }}(a)$ which are obtained by using the functions $a^{\text {out }}\left(x, p^{\text {out }}\right)$ and $a^{\text {in }}\left(x, p^{\text {in }}\right)$. The parameters of the neutron (quark) star are then given by the condition

$$
M_{p_{\text {out }}}^{\text {out }}(a)=M_{p_{\text {in }}}^{\text {in }}(a) .
$$

The condition (2.3) determines $M$ and $a$ precisely, if the resonant frequencies are determined precisely. If an error occurs in determination of the resonant frequencies, as naturally expected, our method gives related intervals of acceptable values of mass and spin parameter of the neutron (quark) star.

\section{Resonant switch model applied to the source $4 \mathrm{U} 1636-53$}

We test the RS model in the case of the atoll $4 \mathrm{U} 1636-53$ source that seems to be the best possibility due to the character of the observational data demonstrating clearly existence of two "resonant points" where the energy switch effect occurs. Using the results of Török (2009), the resonant frequencies determined by the energy switch effect are given in the outer resonant point with frequency ratio $\nu_{\mathrm{U}} / \nu_{\mathrm{L}}=3 / 2$ by the frequency intervals

$$
\nu_{\mathrm{U}}^{\text {out }}=\nu_{\mathrm{U} 0}^{\text {out }} \pm \Delta \nu^{\text {out }}=(970 \pm 30) \mathrm{Hz}, \quad \nu_{\mathrm{L}}^{\text {out }}=\nu_{\mathrm{L} 0}^{\text {out }} \pm \Delta \nu^{\text {out }}=(647 \pm 20) \mathrm{Hz},
$$

and at the inner resonant point with frequency ratio $\nu_{\mathrm{U}} / \nu_{\mathrm{L}}=5 / 4$ there is

$$
\nu_{\mathrm{U}}^{\text {in }}=\nu_{\mathrm{U} 0}^{\text {in }} \pm \Delta \nu^{\text {in }}=(1180 \pm 20) \mathrm{Hz}, \quad \nu_{\mathrm{L}}^{\text {in }}=\nu_{\mathrm{L} 0}^{\text {in }} \pm \Delta \nu^{\text {in }}=(944 \pm 16) \mathrm{Hz} .
$$

We consider the standard specific models of the twin oscillations based on the orbital and epicyclic geodetical frequencies and in the framework of the RS model we determine range of allowed values of spin $a$ and mass $M$ of the neutron star located at the atoll source $4 \mathrm{U} 1636-53$.

The predicted ranges of the neutron star parameters are strongly dependent on the twin modes applied in the RS model. The mass and spin estimates of the RS model have to be confronted with restrictions on the neutron star mass and spin implied by the theoretical models of neutron (quark) stars and the observed rotational frequency of the neutron star at $4 \mathrm{U} 1636-53$. We consider as acceptable upper values $a_{\max } \sim 0.4, M_{\max } \sim 2.5 \mathrm{M}_{\odot}$ and demonstrate that for some of the oscillatory modes used in the RS model the predicted parameters of the neutron star are unacceptable, being too high. Among acceptable $\mathrm{RS}$ models the most promising are those combining the relativistic precession (where $\nu_{\mathrm{U}}=\nu_{\mathrm{K}}, \nu_{\mathrm{L}}=\nu_{\mathrm{K}}-\nu_{r}$ ) and the total precession (where $\nu_{\mathrm{U}}=\nu_{\theta}, \nu_{\mathrm{L}}=\nu_{\theta}-\nu_{r}$ ) frequency relations or their modifications. The switch of the oscillating modes is not given by the resonant phenomena necessarily. The cause could be, e.g., given by the magnetic field, then the Alfvén wave model (Zhang 2004, Zhang et al. 2006) can be relevant.

Acknowledgements. We thank grant GAČR 202/09/0772 and the project CZ.1.07/ 2.3.00/20.0071 "Synergy" supporting international collaboration of the Institute of Physics at SU Opava.

\section{References}

Stuchlík, Z., Kotrlová, A., \& Török, G. 2012, AcA (in press)

Török, G. 2009, $A \mathscr{\xi} A, 497,661$

Zhang, C. M. 2004, A\&A, 423, 401

Zhang, C. M., Yin, H. X., Zhao, Y. H. et al. 2006, MNRAS, 366, 1373 